\section{$\underset{\substack{\text { hommes } \\ \text { \& migrations }}}{ }$}

\section{Hommes \& migrations}

Revue française de référence sur les dynamiques

migratoires

$1310 \mid 2015$

Fashion Mix

\title{
Les jeux du paraître de femmes juives originaires de Tunisie
}

\section{Sylvaine Conord}

\section{(apenEdition \\ Journals}

\section{Édition électronique}

URL : http://journals.openedition.org/hommesmigrations/3165

DOI : 10.4000/hommesmigrations.3165

ISSN : 2262-3353

\section{Éditeur}

Musée national de l'histoire de l'immigration

\section{Édition imprimée}

Date de publication : 1 avril 2015

Pagination : 111-115

ISBN : 978-2-919040-31-5

ISSN : $1142-852 X$

\section{Référence électronique}

Sylvaine Conord, «Les jeux du paraître de femmes juives originaires de Tunisie », Hommes \& migrations [En ligne], 1310 | 2015, mis en ligne le 01 avril 2018, consulté le 03 mai 2019. URL : http:// journals.openedition.org/hommesmigrations/3165; DOI : 10.4000/hommesmigrations.3165 


\section{LES JEUX DU PARAÎTRE DE FEMMES JUIVES ORIGINAIRES DE TUNISIE}

Par SYLVAINE CONORD, maître de conférences en sociologie à l'université Paris-Ouest Nanterre-La Défense, chercheure à Mosaïques, LAVUE (CNRS 7218).

Au début des années 2000, certains cafés de Belleville étaient devenus le point de ralliement d'un groupe étonnant de femmes d'origine juive tunisienne. Leur verbe haut et le cliquetis des bijoux soulignaient leurs tenues choisies pour attirer l'œil. L'enquête sociologique mêlée à la photographie a cherché à comprendre cette mise en scène permanente, et sur quels leviers se fonde la construction de l'image de soi : entre transmission des traditions populaires et jeu avec les visions exotisées que suscite leur apparence vestimentaire.

Alors que j'étais installée à une table du café parisien La Vielleuse ${ }^{1}$ pour mener une enquête sur les cafés du quartier de Belleville $e^{2}$, je remarquai trois femmes d'une soixantaine d'années, élégamment vêtues d'habits aux paillettes dorées et portant une quantité incroyable de bijoux sonores. J'entendais, de ma place, l'entrechoquement des bracelets et des colliers. Leurs cheveux étaient teints au henné, laissant apparaitre des racines blanches. Elles portaient une épaisse couche de fond de teint et avaient un fort embonpoint. La clientèle de la salle du café était en majeure partie composée de femmes semblables à mes voisines. Cette présence dans ces lieux, au fil de mes visites dans le café, s'avéra jour après jour régulière, établie et d'une grande visibilité : installées pour la majorité d'entre elles derrière les vitrines du café, ou sur la terrasse extérieure au printemps et en été, elles offraient aux passants le spectacle de leurs rencontres et de leur apparence grâce à leurs vêtements aux couleurs vives qui ne passaient pas inaperçue. Bavardages, disputes, altercations avec le serveur, éclats de voix, tumultes représentaient autant d'éléments constitutifs de leur quotidien, aisément repérables par les habitants du quartier, les clients delétablissement, les simples promeneurs ou les observateurs. 
Des entretiens recueillis au cours d'une deuxième investigation liée à une recherche doctorale $^{3}$ permirent ensuite, d'une part, d'identifier ces femmes comme étant des retraitées d'origine juive tunisienne et, d'autre part, de reconnaitre ce café comme un lieu de rencontre et de fréquentation central pour une partie de cette communauté. "Vous voyez, même les gens qui viennent de n'importe quel côté, ils demandent La Vielleuse. Ils voient des gens qu'ils n'ont pas vus depuis dix ans. On est des compatriotes, des Tunisiens... Il y a des gens quion n'a pas vus depuis des années et on se retrouve comme ça, par hasard. Ils savent que c'est un café où il y a des Tunisiens. Les gens viennent à Paris, ils viennent nous voir, c'est un lieu de rencontre. Je vous jure, c'est la vérité !... C'est un petit club" (Rebecca).

\section{Les cafés de Belleville, lieux de la reconnaissance}

Depuis trente-cinq ans, ces petits groupes féminins se font et se défont ${ }^{4}$ au gré de leurs humeurs, de leurs affinités ou des problèmes de santé, de deuil, mais, quoi qu'il arrive, subsistent

Le quartier de Belleville

est le premier lieu d'ancrage

en France pour les juifs

tunisiens de cette classe

sociale, un lieu parfois imposé

à ces nouveaux habitants. des années 1950, période de la grande vague d’immigration ${ }^{5}$ durant laquelle la majorité des juifs tunisiens ont quitté leur terre natale : elles étaient à ce moment-là âgées d'une vingtaine d'années. Issues de milieux sociaux défavorisés, elles ont, pour la plupart, habité le quartier Hafsia à Tunis (réputé à leur époque comme un quartier pauvre, comme un sous-quartier de la $\mathrm{Hara}^{6}$ ). En France, elles furent cantinières, ouvrières, emballeuses, couturières..., plusieurs d'entre elles ne savent pas lire. Le départ de la majorité des juifs du sol maghrébin a été vécu par les femmes primo-arrivantes en France (celles rencontrées à La Vielleuse) comme un exil, au sens défini par Chantal Bordes-Benayoun : 'L'exil, en tant qu'expérience sociologique, peut être défini comme une migration forcée d'un endroit où l'on est interdit, ou d'un endroit vécu comme tel, à un autre où l'on se fait accepter ${ }^{7}$." Ce sentiment est aussi à l'origine, à l'arrivée en France, d'une volonté de se regrouper ou du moins de rechercher des lieux où il est possible de retrouver des réseaux communautaires, comme les cafés qui représentaient par ailleurs des "lieux du paraître" et de la reconnaissance.

Le quartier de Belleville est le premier lieu d'ancrage en France pour les juifs tunisiens de cette classe sociale, un lieu parfois imposé à ces nouveaux habitants. Les immeubles de Belleville qu'ils habitèrent en arrivant étaient vétustes, la plupart insalubres et exigus. La grande majorité des femmes rencontrées sur le terrain ne les occupaient plus au moment de l'enquête. Après des années d'inconfort, elles ont fini par déménager vers le haut du XIX arrondissement ou en proche banlieue, contraintes de changer de quartier pour bénéficier d'un habitat social répondant à leurs besoins (et obtenu souvent après plusieurs années d'attente). Mais, chaque jour, malgré des problèmes de santé et de mobilité, en empruntant bus et métro, elles revenaient dans ce quartier où elles semblaient avoir construit leurs racines ${ }^{8}$. On comprend alors leur volonté de se montrer coquettes comme faisaient leurs mères lorsqu'elles allaient elles-mêmes fréquenter les cafés de la place Khalaline à Tunis. Il s'agit d'une mise en scène pour se valoriser et donner une belle image de soi. Elles allaient au café pour "oublier leurs soucis" (problèmes de santé, fils au chômage, divorce, solitude parfois) et avaient besoin à ce titre de se créer un monde d'apparence qui conjurerait le sort d'une vie aux multiples difficultés à surmonter. Elles venaient rejoindre leurs 
amies au café pour échanger des avis sur leurs vêtements, leurs bijoux, les affaires en magasin à ne pas rater et parler, des heures durant, en dialecte judéoarabe. Les échanges commerciaux étant placés au centre de leurs relations ${ }^{9}$, il n'était pas rare que l'une d'entre elles, toujours la même, surnommée "Poupée", présente dans le café des habits neufs ou d'occasion à vendre de manière discrète puisque le directeur du café n'aurait pas autorisé ce commerce. C'est là, dans ce café bellevillois, puis dans les fêtes auxquelles je fus invitée grâce au rôle de photographe qu'elles m'attribuèrent, que je compris le rôle du paraître dans leur manière d'être.

\section{Les héritières de traditions populaires}

Ces femmes tiennent une place marginale et controversée au sein de la communauté juive tunisienne. Elles sont tantôt reconnues comme les gardiennes des traditions judéotunisiennes (par leur pratique d'un dialecte spécifique, la perpétuation des coutumes), tantôt déconsidérées. J'ai pu entendre à plusieurs reprises le type de discours suivant: "Mais vous savez, la communauté juive tunisienne, ce r'est pas elles... Si vous voulez étudier les juifs tunisiens ce n'est pas à Belleville qu'il faut aller... Il faut que je vous explique : à Tunis nous nétions pas mélangés, elles habitaient le quartier le plus pauvre, et d'autres en dehors... Nous ne sommes pas du même milieu..." ou : "Vous étudiez les gens de Belleville? Mais ce n'est pas du tout 'La' communauté juive tunisienne, ce n'est pas représentatif du tout! Moi je vais vous présenter ce qu'est 'La' communauté" (habitant de Sarcelles).

L'attitude des juives tunisiennes, associée à celle d'une classe sociale populaire, déplaisait dans les espaces publics bellevillois. Plusieurs expressions servaient à y qualifier leur comportement : "Elles sont vulgaires", "grossières", "triviales"... "Elles crient", "Elles se disputent tout le temps, s'insultent", "Elles parlent fort", "Elles sont fatigantes", "Elles s'habillent mal, c'est criard"... "Comment faites-vous pour les supporter?" L'appellation "les vieilles", utilisée couramment pour les désigner, était employée sur le ton du mépris, de la moquerie ou, inversement, avec une certaine sympathie. La place et l'image de ces femmes dans la communauté juive tunisienne se jouent dans cette ambivalence.

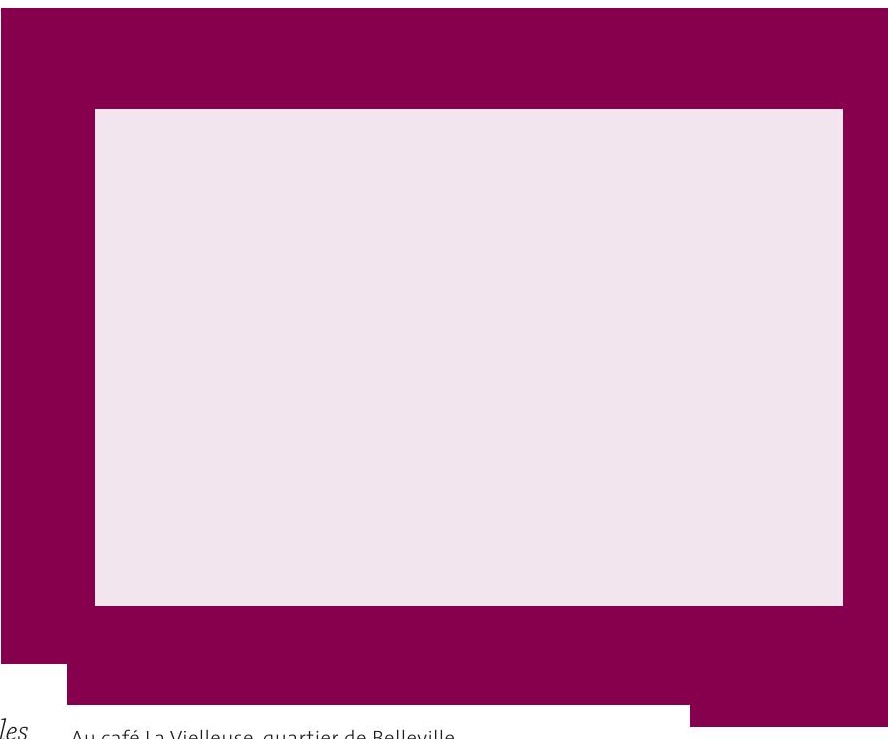

Au café La Vielleuse, quartier de Belleville. (c) SYLVAINE CONORD

D'un côté, leur origine sociale (située en bas de l'échelle sociale d'une société tunisienne fortement hiérarchisée), leurs manières d'être, leur non-respect de certaines pratiques religieuses, leurs croyances à caractère superstitieux et les lieux qu'elles investissent (les cafés), ainsi que leur façon de se vêtir les placent dans la catégorie des milieux sociaux peu fréquentables. Mais, d'un autre côté, leur âge et leur expérience les font reconnaitre comme les héritières des pratiques quotidiennes d'un mode de vie juif tunisien, d'une culture dite "traditionnelle". La 
construction de jugements de valeur se fait à partir des origines sociales et régionales de chaque groupe et la mode en est le reflet. On ne verrait jamais une femme de confession juive très pratiquante porter des pullovers à paillettes ou des robes à l'orientale. Les clivages sociaux existant en Tunisie ont été en partie reproduits en France, du moins à travers les représentations sociales. De plus, une bonne partie des juifs originaires de la Tunisie (bijoutiers, artisans spécialisés, commerçants, etc.) ont dû subir un déclassement à la suite de l'immigration ${ }^{10}$.

\section{Les dangers d'une mise en scène exotisée}

L'approche de cette représentation de l'autre et de cette présentation de soi fut particulièrement prépondérante dans un travail de recherche sociologique où la prise de photographies au centre des interactions était comprise comme un moyen d'investigation ${ }^{11}$. En effet, mes pratiques photographiques étaient admises au café La Vielleuse grâce à la monnaie d'échange que représentaient les images réalisées selon un système de don et contre-don. C'est-à-dire qu'une fois les photos tirées sur papier, je revenais dans le café pour en offrir aux personnes photographiées.

Mes premiers pas sur le terrain furent révélateurs. Fières de leur image, elles n'hésitaient pas à marchander avec moi la moindre prise de vue. Ces femmes avaient une certaine habitude des journalistes audiovisuels et se faisaient payer pour des tournages. Aisément repérables de la rue, leur présence pouvait présenter un caractère original, voire "exotique", par leurs habits (pull-overs, chemisiers, vestes et manteaux) aux couleurs parfois vives, décorés de verroteries rutilantes ou de paillettes dorées, leurs ornements brillants et nombreux (grands colliers de perles, amulettes en forme de

Mme C. posant chez elle en robe "orientale". (c) SYLVAINE CONORD

poisson, bracelets et bagues en or, brillants). Elles pouvaient représenter, d'un point de vue extérieur, un certain folklore subsistant dans un quartier encore réputé comme populaire, mais vivant de profondes et nombreuses mutations urbaines et sociales. Toute la difficulté pour moi, photographe et sociologue, résidait dans les jeux du paraître qui m'entraînaient vers le risque de produire par l'image une vue exotique. Elles-mêmes privilégiaient des auto-mises en scène face à l'appareil, vêtues de leurs plus belles robes, et comprirent par là même qu'il était intéressant de mattribuer un rôle de photographe attitré ${ }^{12}$, les suivant de café en café, de lieu en lieu à Paris, à Juan-les-Pins (surnommé par elle "Belleville plage"), en Tunisie et en Israël lors de pèlerinages. Leur goût du paraître et de la mémorisation de cette image de soi était sans limite. Après avoir rejeté mes images en noir et blanc pour leur caractère "triste" qui ne mettait pas en valeur leurs 
habits colorés, leurs rouges à lèvres ou leurs parures dorées, elles m'imposèrent leur pose (frontale avec un grand sourire) et leur mise en scène. Il s'agissait de bien montrer un style vestimentaire rayonnant que la photographie sert à immortaliser.

Les images tirées sur papier sont destinées à être archivées dans des albums. L'une de ces femmes, Marie $^{13}$, par exemple, m'a commandé plus de 300 images la représentant inlassablement dans différentes tenues face à l'appareil. Il y avait aussi Mme C., plus jeune (47 ans) que ses amies du café, qui m’avait été présentée par son mari. "Ma femme, c'est Madame photo!" m'avait-il prévenue. En effet, Mme C. m'a invitée chez elle pour prendre des photos d'elle habillée de robes à l'orientale, environ vingt dans l'après-midi, toutes plus brillantes les unes que les autres, dans un studio improvisé avec un tissu blanc à fleurs accroché au mur. L'objectif était d'en tirer de très grands formats (50x60) pour les afficher sur les murs de tout son appartement. Mme $C$. est une passionnée de danse orientale et elle voulait recréer par ses auto-mises en scène une image d'elle "à l'orientale". C'est finalement elle qui était à l'origine de cette vision exotique que je voulais éviter dans ma production photographique. Je n'ai vu que chez elle ce genre de robes au décolleté échancré qui brillait de mille feux même quand il y avait peu de lumière.

\section{Production et contrôle de l'image de soi}

Les choix de mise en scène imposés au photographe révèlent en partie la manière dont ces femmes veulent être perçues, montrées, exposées au regard de l'Autre. "En regardant celui qui regarde (ou qui photographie), en rectifiant la tenue, on se donne à regarder comme on entend être regardé, on donne l'image de soi", écrit Pierre Bourdieu ${ }^{14}$. Le procédé photographique devient le moyen de fixer, d'immobiliser, voire d'immortaliser des représentations de soi à travers également l'enregistrement visuel des vêtements que l'on porte et qui deviennent partie de sa personne. David Le Breton remarque : "La photographie personnalise l'homme; en distinguant son corps, et surtout son visage, elle apporte la contribution à la célébration de l'individu ${ }^{15}$."

Les demandes des clientes du café La Vielleuse relatives à la pose ou à l'auto-mise en scène ne furent pas verbalisées de manière précise au moment des prises de vues. Mais la manière dont les corps se positionnaient devant l'appareil, l'expression souriante des visages se figeant à l'instant du déclic, exprimaient déjà cette volonté de contrôler l'image "donnée de soi" et de montrer, de mettre en valeur leurs robes et leurs ornements. Comme le souligne Pierre Bourdieu, "la frontalité est un moyen d'effectuer soi-même sa propre objectivation: donner de soi une image réglée c'est une manière d'imposer les règles de sa propre perception ${ }^{16 "}$. Les entrevues entre la sociologue-photographe et les sujets photographiés montrèrent le besoin de contrôler l'image, ressentie comme celle de soi. "Le portrait accomplit l'objectivation de l'image de soi $i^{17}$." Mais les premières réactions sont aussi révélatrices de ce que David Le Breton évoque à propos de la relation qu'entretient un acteur avec son propre corps, son visage, ou l'écart existant entre "une image de soi, en partie inconsciente, stable au cours de la vie, et l'apparence donnée à voir, soumise au gré des circonstances ${ }^{18}$.

Finalement, pour ces femmes, la construction d'une image de soi passe aussi par la sélection des tissus, des robes, des jupes, des chemisiers, des pullovers, des bijoux qu'elles vont porter, que ce soit pour les fêtes ou la vie courante. Ces choix constamment discutés entre elles rythment leur vie quotidienne. Le portrait photographique ne fait qu'accentuer ces jeux du paraître à travers des mises en scène plutôt normatives (centralité, frontalité, immobilité), car prises dans une dynamique collective. Se vêtir devient une sorte de langage, une communication directe dont l'objectif est d'exister au regard de l'autre. 